\title{
Does increased nuchal translucency indicate a fetal abnormality? A retrospective study to clarify the clinical significance of nuchal translucency in Japan
}

\author{
Shigo Yoshida $\cdot$ Kiyonori Miura $\cdot$ Kentaro Yamasaki · \\ Shoko Miura · Takako Shimada · Terumi Tanigawa • \\ Atsushi Yoshida $\cdot$ Daisuke Nakayama $\cdot$ Hideaki Masuzaki
}

Received: 15 January 2008/Accepted: 21 April 2008/Published online: 24 May 2008

(C) The Japan Society of Human Genetics and Springer 2008

\begin{abstract}
The results of a chromosomal test by genetic amniocentesis in 58 cases with an increased nuchal translucency (NT; $\geq 3 \mathrm{~mm}$ thickness) revealed 47 cases showing a normal karyotype (81\%) and 11 cases (19\%) showing an abnormal karyotype. However, the cases of a normal karyotype with increased NT also included those with fetal abnormalities. Among the 49 cases in which NT was observed during the first trimester and then subsequently disappeared, chromosomal abnormalities were observed in five, and fetal abnormalities other than chromosomal abnormalities were observed in two. Meanwhile, all nine cases in which an increased NT remained or in which NT continued to increase in size during the second trimester were diagnosed as having cystic hygroma, and chromosomal abnormalities were found in six cases (67\%). It should be noted that the shape of increased NT includes NT with a notch (notched NT) and NT without a notch (smooth NT). Among the 20 cases of notched NT, chromosomal abnormalities were observed in eight (40\%), and cystic hygroma was observed in nine (45\%). On the other hand, among the 38 cases of smooth NT, chromosomal abnormalities were observed in three $(7.9 \%)$, but no cystic hygroma was observed. Our results confirm that increased NT does not always indicate a fetal abnormality. Whether NT thickness should be measured as a screening tool for fetal abnormalities remains controversial. However, increased NT may be detected by chance, because a
\end{abstract}

S. Yoshida $\cdot$ K. Miura $(\bowtie) \cdot$ K. Yamasaki $\cdot$ S. Miura

T. Shimada $\cdot$ T. Tanigawa $\cdot$ A. Yoshida $\cdot$ D. Nakayama

H. Masuzaki

Department of Obstetrics and Gynecology,

Nagasaki University Graduate School of Biomedical Sciences,

1-7-1 Sakamoto, Nagasaki 852-8501, Japan

e-mail: kiyonori@nagasaki-u.ac.jp maternal-fetal medical examination using ultrasonography is usually performed in Japan. It is therefore considered to be extremely important to establish a system in which cases are referred to obstetricians who are licensed clinical genetic specialists to obtain appropriate genetic counseling whenever increased NT is clinically observed.

Keywords Nuchal translucency · Prenatal diagnosis · Genetic counseling · Ultrasonography · Obstetrics . Genetic amniocentesis - Screening marker

\section{Introduction}

Nuchal translucency (NT) is a low-intensity area observed in the fetal posterior cervical region upon ultrasonography at 11-14 weeks of gestation. NT itself is a finding inherent in all fetuses and is not necessarily an abnormal finding. The relationship between increased NT and chromosomal abnormalities was first reported by Nicolaides et al. (1992). Since then, the relationship with diseases other than chromosomal abnormalities, such as cardiac, genetic, and urinary system diseases has been described in various reports (Nicolaides et al. 1992; Souka et al. 2005; Westin et al. 2006). However, although there are many reports on the usefulness of NT measurement as a marker for chromosomal abnormalities, maternal age and the degree of increased NT vary in the medical literature, and the frequency of chromosomal abnormalities in cases of increased NT ranges from $11 \%$ to $88 \%$, which also indicates differences. Therefore, no consistent viewpoint has emerged (Pandya et al. 1995; Brambati et al. 1995; Szabo et al. 1995).

It is known that even when increased NT is observed in the first trimester, in most cases, it later spontaneously 
disappears. However, even in cases in which it disappears, the frequency of observing chromosomal abnormalities and other fetal abnormalities has been reported to be higher than that in the general population (Müller et al. 2004). In addition, there are cases in which the increased NT observed during the first trimester lso remains into the second trimester, and it is then diagnosed as cystic hygroma. However, it is difficult to diagnose this abnormality as cystic hygroma during the first trimester.

In Europe and the United States, a One-Stop Clinic for the Assessment of Risk (OSCAR) has been implemented, and a screening test according to maternal age with a serum marker for Down syndrome in addition to NT is conducted. In addition, NT measurement has is one of the items of the screening test for Down syndrome (Bindra et al. 2002).

On the other hand, the situation in Japan is that ultrasonography is almost universally used as part of the normal maternal health checkup. Therefore, whereas the correlation between increased NT and chromosomal abnormalities has been reported in Europe and the United States, NT measurement became widely used in Japan without sufficient understanding of the clinical significance of such measurement and how to accurately interpret such findings. It is therefore common for increased NT to be identified by chance during ultrasonographic examinations, and a serious issue has recently arisen in which artificial abortion often tends to be selected when increased NT is observed in a fetus, because sufficient genetic counseling has not been conducted.

Therefore, to clarify the clinical significance of NT in Japan, we studied the frequency of chromosomal abnormalities in cases of increased NT, the frequency of fetal abnormalities in cases of a normal karyotype with increased NT, the frequency of fetal abnormalities in cases with disappearance of NT, and the relationship between NT shape and fetal abnormalities.

\section{Materials and methods}

Subject

To obtain data of chromosomal karyotype, we included 171 pregnant women who received genetic amniocentesis at 16 weeks of gestation between February 1998 and May 2007 at the Department of Obstetrics and Gynecology at Nagasaki University Hospital. This included 102 cases with advanced maternal age ( $\geq 35$ years old), 58 with NT thickness of at least $3 \mathrm{~mm}$ (increased NT), five with a history of delivering children with chromosomal abnormalities, and six others (four of a request for a chromosomal test, one with a history of delivery with Noonan syndrome, and one with a history of delivering a child with a cardiac abnormality). All cases were managed at regional private clinics and referred to Nagasaki University Hospital for genetic counseling. For all cases, genetic counseling was performed by obstetricians who were licensed clinical genetic specialists. Patient consent before measuring NT was obtained, and NT thickness was measured between 11 and 14 weeks of gestation in 171 cases of single pregnancy.

NT measurement by ultrasonography

NT was measured by an ultrasound specialist according to the method stipulated by the Fetal Medicine Foundation (Nicolaides et al. 1999). NT thickness of $\geq 3 \mathrm{~mm}$ was defined as increased NT, because the frequency of abnormal karyotypes in fetuses with NT thickness $\geq 3 \mathrm{~mm}$ at 11-14 weeks of gestation was higher than the respective number expected on the basis of maternal age (Pandya et al. 1995). Once fetuses with increased NT were detected at 11-14 weeks of gestation, they were followed as cases of increased NT until the end of pregnancy. We measured NT thickness before genetic amniocentesis at 16 weeks of gestation. We also performed a fetal screening of structural abnormalities by ultrasonography at 20 weeks of gestation. When increased NT at 11-14 weeks of gestation was not detected visually or had decreased to $<3 \mathrm{~mm}$ at 16 weeks of gestation, those cases were defined as cases of NT disappearance.

\section{Classification of the shape of increased NT}

Cases in which NT had a smooth surface were defined and classified as smooth NT (s-NT group; Fig. 1a), and those in which the NT surface had a notch-like dent were classified as notched NT (n-NT group; Fig. 1b). The frequency of chromosomal abnormalities and the frequency of cases diagnosed as cystic hygroma in the second trimester were compared between the groups.

Statistical analysis

For statistical analysis, the Mann-Whitney $U$ test was used. A value of $P<0.05$ was determined to indicate a significant difference.

\section{Results}

Frequency of fetal chromosomal abnormalities in fetuses with increased NT

As a result of genetic counseling at our hospital, among the 171 cases in which subjects had undergone genetic 
Fig. 1 Classification according to the shape of nuchal translucency (NT). a NT with a smooth surface was classified as smooth NT, and b NT with a notch (arrow) was classified as notched NT
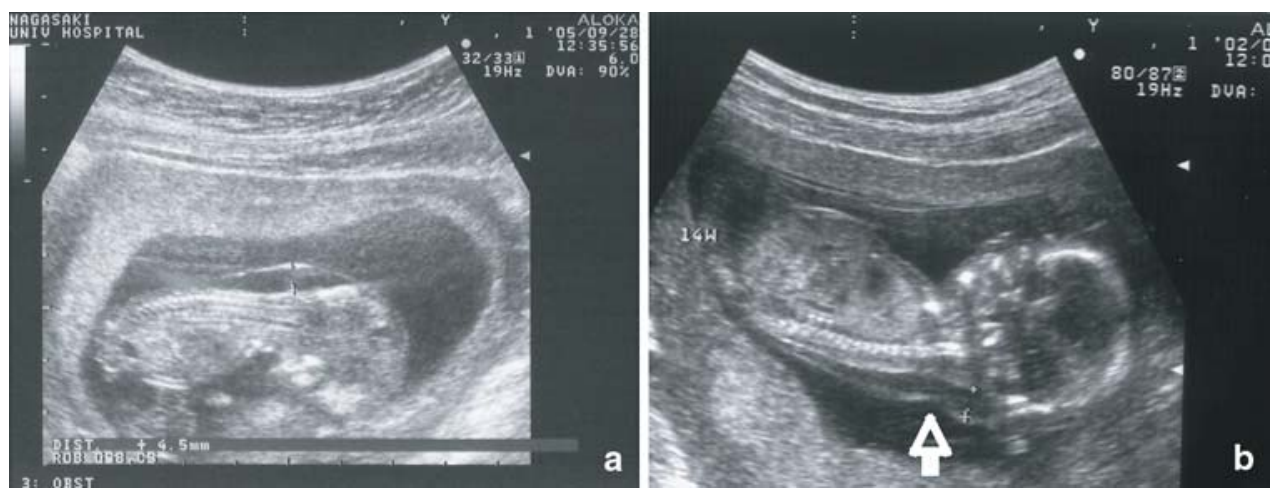

amniocentesis, an increased NT was observed in 58 cases. Among the 58 cases with increased NT, chromosomal abnormalities were observed in 11(19\%), including five of trisomy 21 , for of trisomy 18 , one of monosomy $\mathrm{X}$, and one of mosaic (Fig. 2).

Frequency of chromosomal abnormalities in fetuses demonstrating NT measuring less than $3 \mathrm{~mm}$

The relationship between increased NT and chromosomal abnormalities has been reported, but some cases without an increased NT also have chromosomal abnormalities. Therefore, the frequency of fetal abnormalities was studied in 113 cases with an NT thickness $<3 \mathrm{~mm}$. Indications for undergoing a chromosomal test were advanced maternal age in 102 cases, history of delivery with chromosomal abnormalities in five, history of delivery with Noonan syndrome in one, history of delivering a child with a cardiac abnormality in one, and request for a chromosomal test in four. Among these 113 cases, chromosomal abnormalities were observed in six (5\%), including four of trisomy 21 and two of trisomy 18 . One case of single atrium and single ventricle (1/107 cases; 0.9\%) was detected in 107 fetuses with normal karyotype and normal NT.

\section{Abnormalities in fetuses with normal karyotype and increased NT}

As various fetal abnormalities other than chromosomal abnormalities have been reported for cases with increased NT, we studied which of the possible causes of increased NT were present. The screening of structural abnormalities by ultrasonography was performed at 20 weeks gestation. As a result of the amniotic fluid chromosomal test, among the 58 cases with NT measuring at least $3 \mathrm{~mm}, 47(81 \%)$ demonstrated normal karyotype. Among the 47 normal karyotype cases with NT measuring at least $3 \mathrm{~mm}$, fetal abnormalities were observed in five (5/47 cases; $10.6 \%)$, including one of cardiac abnormality, two of fetal hydrops, one of fetal pleural effusion, and one of diaphragmatic hernia (Fig. 2).

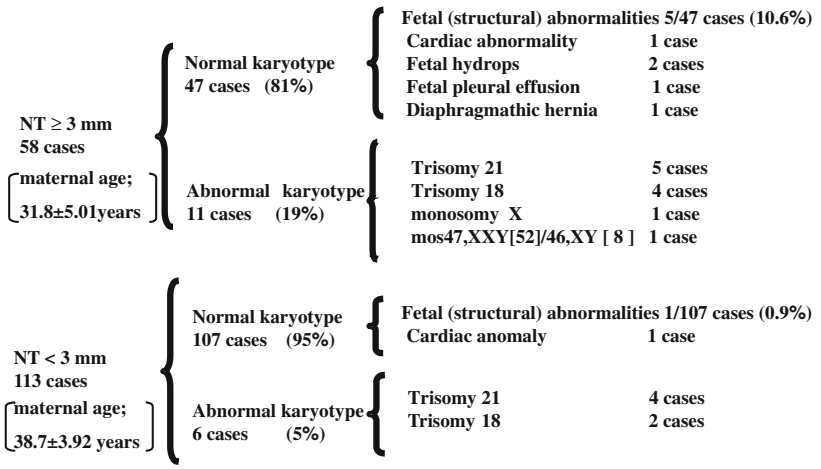

Fig. 2 Nuchal translucency (NT) thickness, fetal chromosomal abnormalities, and fetal structural abnormalities. A flow chart of 58 cases with an increased NT of at least $3 \mathrm{~mm}$ observed in the first trimester and the 113 cases with an NT measuring less than $3 \mathrm{~mm}$

Outcomes in the cases of increased NT that later disappeared

It is known that most cases of increased NT observed in the first trimester disappear as pregnancy progresses. We therefore investigated the frequency of fetal abnormalities in such cases. The average NT thickness in cases of increased NT that later disappeared was $4.1 \pm 1.4(\mathrm{~mm})$. Among the 58 cases in which increased NT was observed in the first trimester, $49(84 \%)$ showed a disappearance of NT in the second trimester. In five of those cases, chromosomal abnormalities (three of trisomy 21 and two of trisomy 18) were observed; and in another two, congenital abnormalities (one each of diaphragmatic hernia and single atrium and single ventricle) were observed (Fig. 3).

Subsequent outcomes in cases of increased NT that did not later disappear

Meanwhile, among the 58 cases in which NT was observed in the first trimester, nine $(16 \%)$ did not show NT disappearance, even during the second trimester. All of these cases were diagnosed to have cystic hygroma, and chromosomal abnormalities were observed in six $(67 \%)$ of the nine cases. The breakdown includes two cases of trisomy 


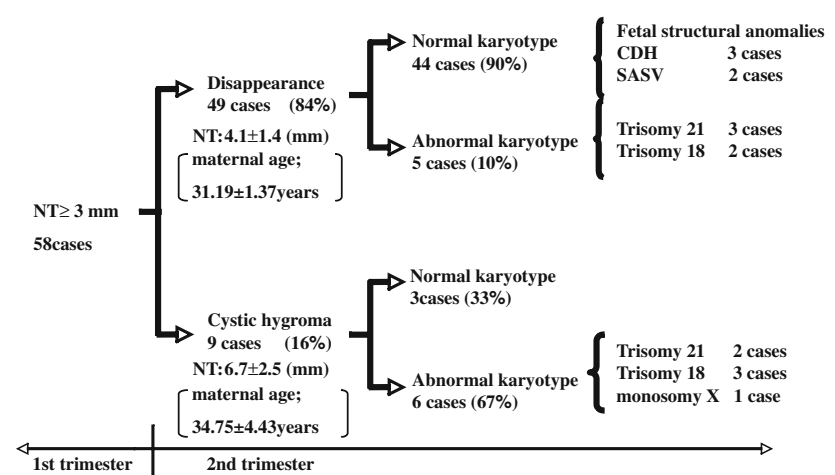

Fig. 3 Outcome of nuchal translucency (NT) observed in the first trimester. The outcomes of 58 cases with increased NT measuring at least a $3 \mathrm{~mm}$ in the first trimester are shown in the flow chart. In the second trimester, increased NT disappeared in 49 cases $(84 \%)$ but remained in nine $(16 \%)$ who were later diagnosed to have cystic hygroma. $C D H$ congenital diaphragmatic hernia, $S A S V$ single atrium and single ventricle

21, three of trisomy 18, and one of monosomy X (Fig. 3). The average NT thickness in the nine cases of cystic hygroma was $6.7 \pm 2.5(\mathrm{~mm})$.

Relationship between NT shape and fetal abnormalities

The average NT thickness in 38 cases of s-NT was $3.9 \pm 1.4(\mathrm{~mm})$, whereas that in 20 cases of $\mathrm{n}-\mathrm{NT}$ was $5.5 \pm 2.2(\mathrm{~mm})$. There was significant difference between the groups $(P<0.0006)$. All nine cases diagnosed as cystic hygroma colli in the second trimester demonstrated increased NT with a notch in the first trimester. Maymon et al. (2001) reported a notch in $62 \%$ of NT cases that showed increased NT in the first trimester and that were later diagnosed with Down syndrome. It was thus indicated that NT with a notch is a marker more closely related to Down syndrome. As described above, because cystic hygroma has a high frequency of being accompanied by chromosomal abnormalities, we analyzed the relationship between NT with a notch observed in the first trimester and chromosomal abnormalities as well as cystic hygroma, and we studied whether NT with a notch observed in the first trimester can be regarded as a marker for fetal abnormalities and cystic hygroma.

As a result of classifying 58 cases of increased NT according to the presence or absence of a notch, the s-NT group consisted of 38 cases, whereas the n-NT group consisted of 20 cases (Fig. 4). In the s-NT group, three cases had chromosomal abnormalities (7.9\%; two of trisomy 21 , one of trisomy 18 ), and in the n-NT group, eight $(40 \%)$ had chromosomal abnormalities. The frequency of chromosomal abnormalities in the n-NT group was significantly higher than that in the s-NT group $(P=0.0027)$. Among the eight cases in the n-NT group with chromosomal abnormalities indicated, six were diagnosed to have

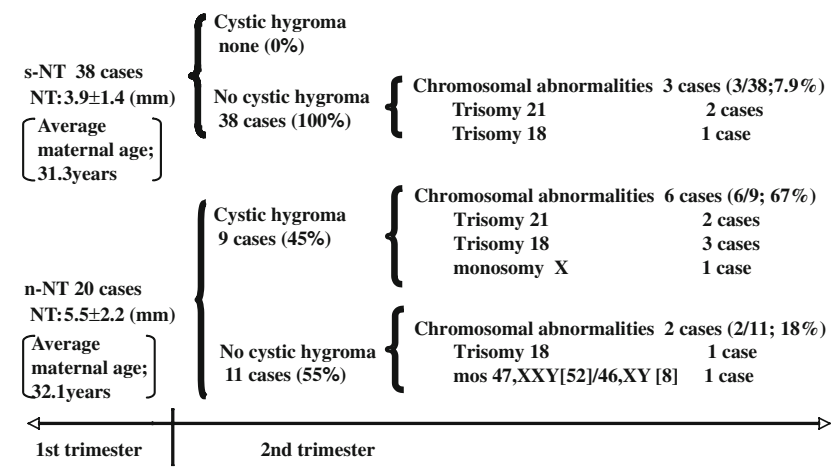

Fig. 4 Relationship between nuchal translucency (NT) patterns and fetal abnormalities. The smooth NT (s-NT) group consisted of 38 cases, whereas the notched NT (n-NT) group consisted of 20 cases. In the s-NT group, three cases $(7.9 \%)$ had chromosomal abnormalities. In the n-NT group, eight cases $(40 \%)$ had chromosomal abnormalities. The frequency of chromosomal abnormalities in the n-NT group was significantly higher than that in the s-NT group $(P=0.0027)$

cystic hygroma in the second trimester, with two of trisomy 21 , three of trisomy 18 , and one of monosomy X. The remaining two were diagnosed as one case each of mosaic (mos 47,XXY[52]/46,XY[8]) and 18 trisomy. In addition, nine cases of cystic hygroma in the second trimester were classified as belonging to the n-NT group, but none were classified as belonging to the s-NT group $(P=0.0001)$.

\section{Discussion}

Among the 58 cases with increased NT, 11 (19\%) had chromosomal abnormalities. The frequencies of chromosomal abnormalities in cases with increased NT varies in reports by different authors, but it was $19.2 \%$ and $16.2 \%$, respectively, in one study of 11,315 cases (Kagan et al. 2006) and one of 1,015 cases (Pandya et al. 1995), and our study results were also similar. Indeed, the frequency of accompanying chromosomal abnormalities is high in cases with increased NT, but conversely, $81 \%$ of the cases with increased NT demonstrate a normal karyotype. This means that most cases are normal karyotype fetuses despite increased NT. The presence or absence of chromosomal abnormalities cannot be determined according to NT thickness, as performing NT measurements is only a screening test. It is therefore necessary to be aware that a chromosomal test is also required to verify a diagnosis of chromosomal abnormalities.

Among 113 cases with NT measuring $<3 \mathrm{~mm}$, chromosomal abnormalities were observed in six $(5 \%)$. As the 113 cases included 102 cases $(90 \%)$ of mothers with advanced maternal age (i.e., $>35$ years of age) and all six cases with chromosomal abnormalities were included in such advanced maternal-age cases, it is believed that chromosomal abnormalities cannot be ruled out, even if NT 
thickness was $<3 \mathrm{~mm}$. Also, the frequency of chromosomal abnormalities is also more significantly affected by maternal age than by increased NT.

As for the outcome of the 47 cases that had increased NT but demonstrated a normal karyotype, fetal abnormalities were observed in five cases (11\%). The breakdown includes two cases of fetal hydrops and one each of cardiac disease, fetal pleural effusion, and diaphragmatic hernia. The reported causes of increased NT include chromosomal abnormalities, heart failure due to cardiovascular and great-vessel anomalies, diaphragmatic hernia, venostasis in the head and neck, abnormal development and outflow obstruction of the lymph system, renal and urinary system, nervous system, genetic disease, and such. Various causes were observed in our study as well. As most instances of NT disappear during the second trimester despite having previously been observed, NT is a thus considered to be a transient physiological finding of the skin during the first trimester, and because it cannot be explained by any single mechanism, it should therefore be used as a collective term in transient findings. Therefore, regarding outcome after detection of NT, NT disappears during the second trimester of pregnancy in some cases but continues to grow in others.

We studied the outcome of 49 cases in which increased NT was observed in the first trimester and disappeared during the second trimester. Among these cases, chromosomal abnormalities (three of trisomy 21 and two of trisomy 18) were observed in five cases, and congenital abnormalities (one case each of diaphragmatic hernia, single atrium, and single ventricle) were observed in two cases. These results indicate that it is not a finding that rules out disease, even when a previously observed increased NT has disappeared, and it is therefore necessary to carefully follow-up such with an increased NT.

The relationship between cystic hygroma and chromosomal abnormalities has been reported, but it is difficult to differentiate NT from cystic hygroma in the first trimester. In addition, no consensus diagnostic criteria have been established for cystic hygroma in the first trimester. It is relatively easy to diagnose so-called multilocular septated cystic hygroma that has septal walls in the fetal posterior cervical region. However, it is very difficult to differentiate nonseptated cystic hygroma without septal walls and an increased NT (Pistorius and Page-Christiaens 2005). In our study, the frequency of chromosomal abnormalities in the $\mathrm{n}$-NT group was significantly higher than that in the s-NT group $(P=0.0027)$. In addition, the nine cases subsequently diagnosed to have cystic hygroma were classified as n-NT, and none were classified as s-NT $(P=0.0001)$. A relationship between n-NT and Down syndrome has been indicated (Maymon et al. 2001), and the cases with cystic hygroma had a high frequency of chromosomal abnormalities $(67 \%)$ in our study, suggesting a positive relationship between. The increased NT observed in the first trimester includes the initial findings of cystic hygroma in the second trimester, and it was indicated that such cases tend to have a notch in NT. The average NT thickness in nine cases of cystic hygroma was significantly increased compared with that in cases of increased NT that later disappeared $(6.7 \pm 2.5 \mathrm{~mm}$ vs. $4.1 \pm 1.7 \mathrm{~mm}$, respectively; $P=0.002$ ). There was also significant difference in NT thickness between the s-NT group and the n-NT group $(P<0.0006)$. Further large-scale studies will clarify the association between NT thickness in the first trimester and disappearance of increased NT in the second trimester or cystic hygroma in the second trimester. The association between fetal abnormalities and NT disappearance or shape in fetuses with normal karyotype is interesting, though the sample numbers in the our study were too small to give sufficient strength to the analysis. Further study regarding this association should be performed.

In Europe and the United States, a screening system for chromosomal abnormalities has been established that integrates and determines maternal age, and maternal serum-marker tests results in addition to NT measurements while calculating the probability of Down syndrome. NT measurements are handled in a similar way as other maternal serum markers. Therefore, clinical fetal chromosomal abnormalities and fetal structural abnormalities are never evaluated by NT measurements alone.

Conversely, in Japan, maternal serum markers were introduced as a screening test for Down syndrome in the early 1990s, and it became widely used because of its simplicity without fully understanding that this test is only a screening test. Because a system of genetic counseling had at that time not been developed, the explanation of the test results was therefore insufficient, and a situation occurred in which couples sometimes elect to have an artificial abortion before undergoing genetic amniocentesis, which would verify such a diagnosis. Therefore, a negative opinion of the implementation of maternal serum markers was issued by the Health Sciences Council in 1999 in Japan.

In addition, regarding NT measurement, a similar problem to that of maternal serum markers has recently been identified. In Japan, NT can be easily measured because fetal ultrasonography is normally performed as part of the regular maternal health checkup. Therefore, these measurements alone became widely used before the clinical significance of NT measurement was fully understood. As a result, we are now struggling with the interpretation and explanation of NT in Japan. In addition, because NT can be observed in images and is indicated as a numeric value, the anxiety that parents feel may be greater than that for the results of serum markers. Parents of a fetus 
with an increased NT may elect to have an artificial abortion without pursuing a confirmation of diagnosis via amniotic fluid testing before sufficiently understanding the significance of NT.

In conclusion, our results confirmed that increased NT does not always indicate a fetal abnormality. As NT measurement is only a screening test and not verification of any diagnosis, normal fetuses with increased NT should not be artificially aborted. Genetic counseling including information regarding both NT and genetic amniocentesis should be performed in all cases before and after measuring NT. Whether NT thickness should be measured as screening of fetal abnormalities remains controversial in Japan. However, increased NT may be detected by chance, because a maternal-fetal medical examination using ultrasonography is usually performed in Japan. It is therefore considered to be extremely important to establish a system in which all cases of increased NT are referred to obstetricians who are licensed clinical genetic specialists to obtain appropriate genetic counseling regarding NT.

Acknowledgments KM was supported in part by Seeds (No.J079500122) from Japan Science and Technology Agency (JST), Grants from The Naito Foundation and Grants-in-Aid for Scientific Research (No.19791155) from the Ministry of Education, Sports, Culture, Science and Technology of Japan.

\section{References}

Bindra R, Heath V, Liao A, Spencer K, Nicolaides KH (2002) Onestop clinic for assessment of risk for trisomy 21 at 11-14 weeks: a prospective study of 15030 pregnancies Ultrasound Obstet Gynecol 20:219-225
Brambati B, Cislaghi C, Tului L, Alberti E, Amidani M, Colombo U, Zuliani G (1995) First-trimester Down's syndrome screening using nuchal translucency: a prospective study in patients undergoing chorionic villus sampling. Ultrasound Obstet Gynecol 5:9-14

Kagan KO, Avgidou K, Molina FS, Gajewska K, Nicolaides KH (2006) Relation between increased fetal nuchal translucency thickness and chromosomal defects. Obstet Gynecol 107:6-10

Maymon R, Dreazen E, Buckovsky I, Weinraub Z, Herman A (2001) Does a 'notched' nuchal translucency indicate Down syndrome fetuses or other adverse pregnancy outcome? Prenat Diagn 21:403-408

Müller MA, Pajkrt E, Bleker OP, Bonsel GJ, Bilardo CM (2004) Disappearance of enlarged nuchal translucency before 14 weeks' gestation: relationship with chromosomal abnormalities and pregnancy outcome. Ultrasound Obstet Gynecol 24:169-174

Nicolaides KH, Sebire NJ, Snijders R (1999) The 11-14-week scan: the diagnosis of fetal abnormalities. Parthenon Publishing, Lancaster

Nicolaides KH, Azar G, Byrne D, Mansur C, Marks K (1992) Fetal Nuchal translucency: ultrasound screening for chromosomal defect in first trimester of pregnancy BMJ 304:867-869

Pandya PP, Kondylios A, Hilbert L, Snijders RJ, Nicolaides KH (1995) Chromosomal defects and outcome in 1015 fetuses with increased nuchal translucency. Ultrasound Obstet Gynecol 5:15-19

Pistorius LR, Page-Christiaens GC (2005) First-trimester septated cystic hygroma: prevalence, natural history, and pediatric outcome. Obstet Gynecol 106:288-294

Souka AP, Von Kaisenberg CS, Hyett JA, Sonek JD, Nicolaides KH (2005) Increased Nuchal translucency with normal karyotype. Am J Obstet Gynecol 192:1005-1021

Szabo J, Gellen J, Szemere G (1995) First-trimester ultrasound screening for fetal aneuploidies in women over 35 and under 35 years of age. Ultrasound Obstet Gynecol 5:161-163

Westin M, Saltvedt S, Bergman G, Almström H, Grunewald C, Valentin L (2006) Is measurement of nuchal translucency thickness a useful screening tool for heart defects? A study of 16838 fetuses. Ultrasound Obstet Gynecol 27:632-639 